\title{
ARDS in an ex-premature infant with bronchopulmonary dysplasia and COVID-19
}

\author{
Meena Kalyanaraman ${ }^{1}$, Derrick McQueen ${ }^{1}$, Kavita Morparia $^{1}$, and Maria Bergel ${ }^{1}$ \\ ${ }^{1}$ Newark Beth Israel Medical Center
}

June 12,2020

\begin{abstract}
SARS-CoV-2 infection has caused a pandemic which has resulted in more severe disease in adults than children. We report an ex-premature infant with bronchopulmonary dysplasia who developed COVID-19 associated severe acute respiratory distress syndrome causing bradycardic cardiac arrest and seizures.
\end{abstract}

Title: ARDS in an ex-premature infant with bronchopulmonary dysplasia and COVID-19 Author information:

Meena Kalyanaraman, $\mathrm{MD}^{1}$; Derrick McQueen $\mathrm{MD}^{1}$; Kavita Morparia $\mathrm{MD}^{1}$; Maria Bergel $\mathrm{MD}^{1}$

Author affiliation:

1: Pediatric Critical Care Medicine

Children's Hospital of New Jersey at Newark Beth Israel Medical Center

Corresponding author:

Meena Kalyanaraman, MD

Associate Director, Pediatric Critical Care Medicine

Children's Hospital of New Jersey at Newark Beth Israel Medical Center

C-5, 201 Lyons Ave

Newark NJ 07112

Phone: 862-763-1742

Email: mkalyanaraman68@gmail.com; Meena.Kalyanaraman@rwjbh.org

Fax: 973-926-6452

Financial assistance : None Conflicts of interest : None

Key words not in title : hypoxemia, cardiac arrest, seizures, ventilation, prone

ARDS in an ex-premature infant with bronchopulmonary dysplasia and COVID-19

Abstract : SARS-CoV-2 infection has caused a pandemic which has resulted in more severe disease in adults than children. We report an ex-premature infant with bronchopulmonary dysplasia who developed COVID-19 associated severe acute respiratory distress syndrome causing bradycardic cardiac arrest and seizures. 


\section{Case report :}

Our institutional review board waived informed consent for publication of this case. A four month old male infant with bronchopulmonary dysplasia (BPD) born premature at 28 weeks gestation with very low birth weight of $1.315 \mathrm{~kg}$, whose corrected age is 1 month, presented with severe respiratory distress and seizures. He was on non-invasive positive pressure ventilation for 90 days for respiratory distress syndrome at birth complicated by human metapneumovirus pneumonia and apnea. Patient had been sick for 5 days prior to admission with cough, nasal congestion and increased work of breathing. He was cared for by his parents who were sick with coronavirus disease (COVID-19) symptoms.

Patient presented to the emergency department of a referral hospital lethargic, seizing and hypoxemic with saturation by pulse oximetry of $77 \%$. There was no history of fever, vomiting, diarrhea, rash or decrease in oral intake. He deteriorated and had a bradycardic cardiac arrest and was resuscitated. Initial arterial blood gas analysis was pH 7.20/pCO2 $81 \mathrm{~mm} \mathrm{Hg} / \mathrm{pO} 239 \mathrm{~mm} \mathrm{Hg} /$ bicarbonate $25 \mathrm{mEq} / \mathrm{L} /$ base excess -6 on 1.0 fraction of inspired oxygen ( $\mathrm{FiO} 2$ ) with $\mathrm{PaO} 2 / \mathrm{FiO} 2$ ratio of 39 and oxygenation index of 41 . Blood and urine cultures were obtained, broad spectrum antibiotics and levetiracetam were started, fluid boluses were given and chest radiograph (CXR) revealed bilateral opacities with central predominance.

Upon arrival in our pediatric intensive care unit (PICU) he was placed on the ventilator using synchronized intermittent mandatory ventilation/pressure regulated volume control mode with FiO2 1.0, positive end expiratory pressure $10 \mathrm{~cm}$ water, tidal volume 55 , Rate $40 /$ minute, inspiratory time $0.7 \mathrm{sec}$ to maintain saturations greater than 90\%. Patient was sedated and placed on neuromuscular blockade. CXR showed significant worsening of bilateral infiltrates. (Figure) Within 24 hours of hospitalization patient became more hypoxemic, inhaled nitric oxide was started and within 36 hours patient's oxygen saturation decreased to the 70 s and patient was proned. Saturations improved and FiO2 was weaned to 0.40 within 8 hours of proning. He was proned for 12-14 hours each day for 4 days.

Rapid real-time reverse transcription polymerase chain reaction test was positive for COVID-19 on nasopharyngeal swab specimen and respiratory viral pathogen panel did not reveal any other viral co-infections. Initial white cell count was $19.6 \times 10^{3} / \mathrm{mcL}$ with $44 \%$ segmented neutrophils, $1 \%$ bands, $48 \%$ lymphocytes, and $6 \%$ monocytes. Hemoglobin was $10.5 \mathrm{~g} / \mathrm{dL}$ and platelet count was $401 \mathrm{x} 10^{3} / \mathrm{mcL}$ which decreased in a few hours to $115 \times 10^{3} / \mathrm{mcL}$. Prothrombin time (PT), partial thromboplastin time (PTT) and international normalized ratio (INR) were slightly increased at 13.9 seconds, 1.41 and 26.8 seconds respectively. Patient received packed red blood cell transfusion, fresh frozen plasma and vitamin K. C-reactive protein was elevated at $3.68 \mathrm{mg} / \mathrm{dL}$ and procalcitonin was high at $0.88 \mathrm{ng} / \mathrm{ml}$. Blood urea nitrogen was $25 \mathrm{mg} / \mathrm{dL}$, serum creatinine was $0.35 \mathrm{mg} / \mathrm{dL}$ and serum sodium was $126 \mathrm{mmol} / \mathrm{L}$ and they improved with volume resuscitation. Alanine transaminase (ALT) and aspartate transaminase (AST) increased significantly from $<120$ unit/L $(\mathrm{U} / \mathrm{L})$ and $<60 \mathrm{U} / \mathrm{L}$ to peak of $1505 \mathrm{U} / \mathrm{L}$ and $2297 \mathrm{U} / \mathrm{L}$ respectively within 12 hours of hospitalization . These enzymes slowly decreased over a period of 3 weeks to near normal values. Albumin decreased from $3.8 \mathrm{~g} / \mathrm{dL}$ on admission to $2.1 \mathrm{~g} / \mathrm{dL}$ in 24 hours . Video electroencephalogram did not show seizures, head ultrasound revealed normal study. Echocardiogram showed normal biventricular systolic function. Patient received methylprednisolone and hydroxychloroquine. Remdesivir was not approved by the manufacturer secondary to severe transaminitis. Patient was extubated on hospital day (HD) 8. Patient remained on non - invasive positive pressure ventilation until HD 22 . He was on supplemental oxygen via nasal cannula for another five days and discharged to home on albuterol and budesonide nebulizers, chlorothiazide, spironolactone, and levetiracetam. Patient was playful and interactive at the time of discharge from our hospital.

\section{Discussion}

We report for the first time to our knowledge a case of COVID-19 acute respiratory distress syndrome (ARDS) causing severe hypoxemia leading to bradycardic cardiac arrest and seizures in an ex-premature infant with comorbidity of bronchopulmonary dysplasia.

After the first detection of novel coronavirus 2019 (SARS-CoV-2) in China in early December 2019 initial 
studies showed most children were asymptomatic or had mild and moderate symptoms. (1) The percentage of severe and critical cases in the pediatric patients with COVID-19 was 5.9\% compared to $18.5 \%$ in adult patients. Admission rates to PICU range from 9.7\% among infected children to $20 \%$ of the hospitalized patients. (2,3) ARDS has been reported in 3-5.8\% of all COVID19 patients and in about $60 \%$ of patients requiring ICU care in adults and children. Our patient met the Berlin criteria for severe ARDS with $\mathrm{PaO} 2 / \mathrm{FiO} 2$ ratio $<100$. Our patient had worsening hypoxemia and increased inflammatory markers of CRP and procalcitonin, low platelets and initial acute kidney injury similar to that seen by Chao and colleagues. (4) The severe transaminitis noted in our patient has not been reported in the pediatric studies published thus far. Our patient had several risk factors for impaired alveolarization and normal lung growth after birth secondary to the prolonged need for oxygen, non-invasive positive pressure ventilation and postnatal infection with human metapneumovirus. (5) The abnormal lung development in our patient put him at high risk for ARDS from COVID-19 that led to hypoxemic acute respiratory failure, bradycardic cardiac arrest and seizures. In conclusion, the various manifestations of COVID-19 in children are still not entirely known. Pediatricians and pediatric critical care physicians should be cognizant of the development of ARDS from SARS-CoV-2 infections in patients with comorbidity of prematurity and associated chronic lung disease as the various factors that interfere with their post natal lung development continue to be studied.

\section{References:}

1. Dong Y, Mo X., Hu Y et al. Epidemiological characteristics of 2143 pediatric patients with 2019 coronavirus disease in China. Pediatrics. 2020; https://doi.org/10.1542/peds.2020-0702

2. Tagarro A, Epalza C, Santos M, et al. Screening and Severity of Coronavirus Disease 2019 (COVID-19) in Children in Madrid, Spain [published online ahead of print, 2020 Apr 8]. JAMA Pediatr. 2020;10.1001/jamapediatrics.2020.1346. doi:10.1001/jamapediatrics.2020.1346

3. DeBiasi RL, Song X, Delaney M et al. Severe COVID-19 in Children and Young Adults in the Washington, DC Metropolitan Region. J Peds. May 2020. DOI: https://doi.org/10.1016/j.jpeds.2020.05.007

4. Chao JY, Derespina KR, Herold BC et al. Clinical Characteristics and Outcomes of Hospitalized and Critically Ill Children and Adolescents with Coronavirus Disease 2019 (COVID-19) at a Tertiary Care Medical Center in New York City. J Peds May 2020. DOI: https://doi.org/10.1016/j.jpeds.2020.05.006

5. O'Reilly M, Sozo F, Harding R. Impact of preterm birth and bronchopulmonary dysplasia on the developing lung: long-term consequences for respiratory health. Clin Exp Pharmacol Physiol. 2013; 40: 765-773.

\section{Figure legend}

Figure: Multifocal bilateral lung infiltrates 


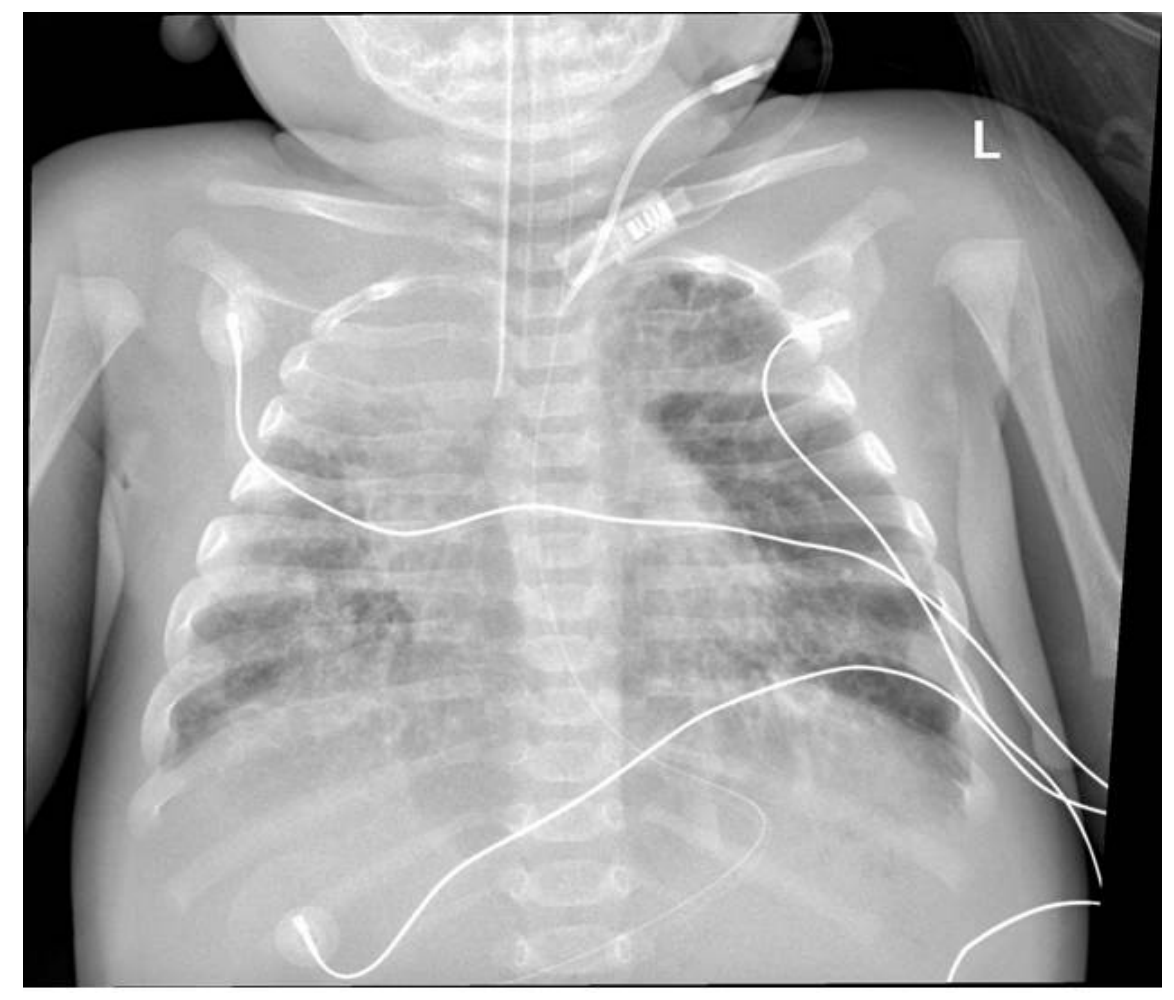

